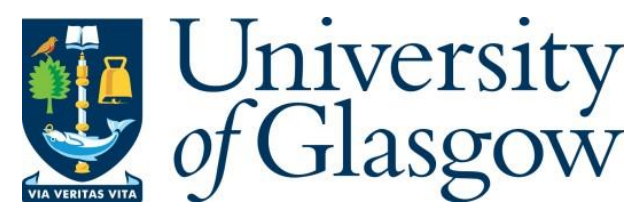

Kakitani, M. T., Brante, G., Souza, R. D., and Imran, M. A. (2013) Downlink Energy Efficiency Analysis of Some Multiple Antenna Systems. In: 2013 IEEE 77th Vehicular Technology Conference (VTC Spring), Dresden, Germany, 02-05 Jun 2013, ISBN 9781467363372.

There may be differences between this version and the published version. You are advised to consult the publisher's version if you wish to cite from it.

$\underline{\text { http://eprints.gla.ac.uk/136779/ }}$

Deposited on: 13 February 2017

Enlighten - Research publications by members of the University of Glasgow http://eprints.gla.ac.uk 


\title{
Downlink Energy Efficiency Analysis of Some Multiple Antenna Systems
}

\author{
Marcos Tomio Kakitani*†, Glauber Brante*, Richard Demo Souza*, and Muhammad Ali Imran ${ }^{\dagger}$ \\ *CPGEI, Federal University of Technology - Paraná, Curitiba, Brazil \\ ${ }^{\dagger}$ CCSR, University of Surrey, Guildford, UK \\ mtkakitani@ieee.org, gbrante@ieee.org, richard@utfpr.edu.br, m.imran@surrey.ac.uk
}

\begin{abstract}
In this paper we compare the energy efficiency of different multiple antenna transmission schemes for longrange wireless networks, assuming a realistic power consumption model. We consider the downlink, between a base station and a mobile station, in which the Alamouti scheme, transmit beamforming, receive diversity, spatial multiplexing, and transmit antenna selection are compared. Our analysis shows that, for different types of base stations, outage probability requirements and spectral efficiencies, the transmit antenna selection scheme is in general the most energy efficient option. Although antenna selection is not the best in terms of outage probability, it becomes the most efficient in terms of overall power consumption as it requires a single radio-frequency chain to obtain spatial diversity.
\end{abstract}

\section{INTRODUCTION}

Energy efficiency has become one of the main concerns of the wireless network operators. Such increased concern can be justified by two main reasons: ecological and economical interests. Regarding the ecological issue, the information and communication technology (ICT) industry represents about $2 \%$ of the global $\mathrm{CO}_{2}$ emissions, with the mobile networks operation representing around $10 \%$ of the ICT industry emissions [1]. From the economical point of view, the rising energy costs and also the operational costs of the base stations (BSs) led the network operators to an emerging concern with respect to the energy efficiency. It has been reported that the ICT industry is responsible for $10 \%$ of the energy consumption in the world, with the mobile communication networks alone representing as much as $0.5 \%$ of the global energy consumption [2]. Considering the increased demand for cellular data traffic, with forecasts ranging between a hundredfold to thousandfold increment before 2020 [3], we can conclude that the required expansion of the cellular networks in the near future will reflect both in a significant energy consumption and $\mathrm{CO}_{2}$ emissions increase.

Due to the above critical impacts, the energy consumption of large wireless communication systems has recently attracted the attention of many authors, as [2]-[8]. A BS power consumption model, as presented in [8], needs to include many additional aspects that are not usually considered in modeling simpler devices, as the nodes from a wireless sensor network [9]. For instance, it is showed in [8] that considering realistic power models, the cooling energy consumption of a BS can represent a significant portion of the total energy consumption. Even the power amplifier module positioning has impact on the energy consumption of the BS [8].

Multiple antennas (MIMO) systems can present a considerable signal-to-noise ratio (SNR) improvement if compared to single antenna (SISO) systems. For the same outage requirement, a MIMO system may demand less transmit power. The SNR gains of MIMO schemes for a given target data rate for cellular networks were analyzed in [10]. But the authors only consider the transmit power, and other BS consumption factors as circuitry and cooling are not included in their analysis. In [9] it is shown that if more realistic power models are considered, the advantage of MIMO over SISO is not always evident for short-range communication systems, as wireless sensor networks. However, power models for cellular systems [8] are considerably different than the power models for wireless sensor nodes [9]. The work in [11] showed that for realistic BS power consumption models, MIMO techniques can be less efficient when compared to SISO. However, the authors consider only the case of a spatial multiplexing MIMO system, where the multiple antennas are used for increased spectral efficiency and not for spatial diversity. In [12] we investigated the limiting distances up to which transmit antenna selection outperforms beamforming in terms of energy efficiency for any number of transmit antennas and a single receive antenna.

In this paper we compare the energy efficiency of SISO and MIMO schemes for a target outage probability in large-range wireless networks. We consider schemes in which either the BS, the mobile station (MS), or both, can be equipped with multiple antennas. Spatial diversity and spatial multiplexing MIMO systems are considered, including the vertical Bell Laboratories layered space-time (V-BLAST) scheme, transmit antenna selection (TAS), receive diversity by means of maximum ratio combining (MRC), the Alamouti scheme, and transmit beamforming (BF). Our results show that although $\mathrm{BF}$ presents the best SNR performance in terms of the outage probability, when realistic power consumption models are considered, the TAS scheme is the most energy efficient option. Such energy efficiency advantage of the TAS scheme comes from the fact that only a single radio-frequency (RF) chain is used at the transmitter, while other MIMO schemes use a RF chain per transmit antenna, compromising their energy efficiency.

The rest of this paper is organized as follows. The system model is introduced in Section II. The outage probability and energy efficiency of the different transmission schemes 
considered in this paper are presented in Section III, which are then numerically evaluated in Section IV. Finally, Section V concludes the paper.

\section{System Model}

We investigate the energy consumption per bit given a target outage probability, considering the downlink between a BS and a MS. The BS power model follows [8], where the total energy consumption per bit can be generalized to a linear model, so that

$$
E_{b t}=\frac{N_{T R X} \cdot P_{0}+\Delta_{p} \cdot \mathcal{P}}{R_{b}}
$$

where $N_{T R X}$ represents the number of transceivers (TRXs) or $\mathrm{RF}$ chains (with each of them serving one transmit antenna) of the BS, $P_{0}$ corresponds to the non-load-dependent power consumption at the minimum non-zero output power, $\Delta_{p}$ is the slope of the load-dependent power consumption, $\mathcal{P}$ represents the RF output power at the antenna elements, and $R_{b}$ is the bit rate in bits/s. Furthermore, $R_{b}=\delta \cdot B$, where $\delta$ represents the spectral efficiency, and $B$ is the system bandwidth. As the power consumption of the MS is not relevant compared to the power consumption of the BS, it is not included in the following analysis.

Considering $M_{t}$ transmit and $M_{r}$ receive antennas, the complex $M_{r}$-dimensional received message at the MS is

$$
\mathbf{y}=\sqrt{\mathcal{P} \cdot \gamma} \mathbf{H} \mathbf{x}+\mathbf{n}
$$

where $\gamma$ is to the path loss between the BS and the MS, $\mathbf{H}$ is the $M_{r} \times M_{t}$ matrix of fading gains $h_{i j}$ representing the unity variance Rayleigh quasi-static fading from a transmit antenna $i$ to a receive antenna $j, \mathbf{x}$ is the $M_{t}$-dimensional and unitary energy transmitted message, and $\mathbf{n}$ is the complex $M_{r}$-dimensional AWGN vector, with variance $N_{0} / 2$ per dimension, where $N_{0}$ is the thermal noise power spectral density per Hertz. The path loss between the BS and the MS is [13]

$$
\gamma=\frac{\lambda^{2}}{(4 \pi)^{2} d^{\alpha}}
$$

where $\lambda$ is the wavelength, $d$ represents the distance between the $\mathrm{BS}$ and the MS, and $\alpha$ is the path loss exponent. We consider the path loss after the power amplifier module, thus the antennas power consumption is already included in the power model. The instantaneous SNR in the BS-MS link is

$$
\mathrm{SNR}=\|\mathbf{H}\|^{2} \cdot \rho
$$

where $\rho=\frac{\gamma \cdot \mathcal{P}}{N}$ is the average SNR, and $N=N_{0} \cdot B$ is the noise power.

The following analysis is based on the outage probability, which is defined as the probability that in the transmission from a BS to a MS, the instantaneous SNR falls below the threshold $\beta=2^{\delta}-1$ at the MS [13].

\section{TRANSMISSION SCHEMES}

Next, due to practical constraints, we assume that $M_{t} \in$ $\{1,2\}$ and $M_{r} \in\{1,2\}$. Moreover, we consider the following schemes: single transmit and receive antennas (SISO); two transmit antennas using the Alamouti scheme and one receive antenna (Alamouti 2x1); two transmit antennas applying TAS and one receive antenna (TAS $2 \times 1$ ); one transmit antenna and two receive antennas applying MRC (MRC 1x2); two transmit antennas using the Alamouti scheme and two receive antennas applying MRC (Alamouti+MRC 2x2); two transmit antennas applying TAS and two receive antennas applying MRC (TAS+MRC 2x2); two transmit antennas using BF and two receive antennas applying $\mathrm{MRC}(\mathrm{BF}+\mathrm{MRC} 2 \mathrm{x} 2)$; and finally two transmit antennas and two receive antennas using the V-BLAST scheme (V-BLAST 2x2).

Note that, as $P_{0}$ and $\Delta_{p}$ are fixed, and $R_{b}$ is often also a fixed design parameter, in order to minimize the total energy consumption per bit as defined in (1), we must minimize the required transmit power given a target outage probability for each of the above schemes. Next we determine such minimum required transmit power for the schemes under analysis.

\section{A. SISO}

In the SISO scheme, for which $N_{T R X}=1$, the outage probability is [14]

$$
\mathcal{O}_{S I S O}=1-\exp \left(\frac{-\beta}{\rho}\right) .
$$

Therefore, given a target outage probability $\mathcal{O}^{*}$, the minimum required transmit power $\mathcal{P}=\mathcal{P}_{S I S O}^{*}$ is

$$
\mathcal{P}_{S I S O}^{*}=\frac{-N \beta}{\gamma \ln \left(1-\mathcal{O}^{*}\right)} .
$$

\section{B. Alamouti $2 \times 1$}

In a system employing the Alamouti space-time block code $\left(N_{T R X}=2\right)$ with only one receive antenna, the outage probability can be written as [15]

$$
\mathcal{O}_{A l a(2 \times 1)}=1-\left(1+\frac{2 \beta}{\rho}\right) e^{\frac{-2 \beta}{\rho}} .
$$

The minimum transmit power $\mathcal{P}=\mathcal{P}_{A l a(2 x 1)}^{*}$ for a target outage probability $\mathcal{O}^{*}$ is thus

$$
\mathcal{P}_{A l a(2 \times 1)}^{*}=\frac{-2 \beta N}{\left[\mathcal{W}\left(\left(\mathcal{O}^{*}-1\right) e^{-1}\right)+1\right] \gamma},
$$

where $\mathcal{W}(\cdot)$ is the inverse function of $f(w)=w e^{w}$.

\section{TAS $2 \times 1$}

In the TAS $2 x 1$ scheme, only one out of two transmit antennas is selected during each transmission, thus only a single $\mathrm{RF}$ chain is required $\left(N_{T R X}=1\right)$. The outage probability in this case is [16]

$$
\mathcal{O}_{T A S(2 \times 1)}=\left(1-e^{\frac{-\beta}{\rho}}\right)^{2} .
$$


For a target outage probability $\mathcal{O}^{*}$, the minimum required transmit power is determined as

$$
\mathcal{P}_{T A S(2 \times 1)}^{*}=\frac{-\beta N}{\ln \left(1-\sqrt{\mathcal{O}^{*}}\right) \gamma} .
$$

\section{D. $M R C 1 \times 2$}

In the MRC 1x2 scheme the BS has one antenna and the MS is equipped with two antennas, thus we have spatial diversity only at the receiver, so that $N_{T R X}=1$. The outage probability is given by [17]

$$
\mathcal{O}_{M R C(1 \times 2)}=1-\left(1+\frac{\beta}{\rho}\right) e^{\frac{-\beta}{\rho}}
$$

The minimum required transmit power for a given outage probability is

$$
\mathcal{P}_{M R C(1 \times 2)}^{*}=\frac{-\beta N}{\left[\mathcal{W}\left(\left(\mathcal{O}^{*}-1\right) e^{-1}\right)+1\right] \gamma} .
$$

\section{E. Alamouti + MRC $2 \times 2$}

As both the BS and the MS are equipped with two antennas $\left(N_{T R X}=2\right)$, it is possible to consider the combination of transmit and receive spatial diversity techniques. In the Alamouti+MRC 2x2 scheme, the Alamouti code is employed at the BS and MRC at the MS. The outage probability for this scenario is [15]

$$
\begin{aligned}
\mathcal{O}_{A l a(2 \times 2)} & =\frac{\Gamma\left(4, \frac{2 \beta}{\rho}\right)}{\Gamma(4)} \\
& =1-\left[1+2\left(\frac{\beta}{\rho}\right)+2\left(\frac{\beta}{\rho}\right)^{2}+\frac{4}{3}\left(\frac{\beta}{\rho}\right)^{3}\right] e^{\left(-2 \frac{\beta}{\rho}\right)} .
\end{aligned}
$$

The minimum required transmit power for an outage probability $\mathcal{O}^{*}$ can be found as

$$
\mathcal{P}_{A l a(2 \times 2)}^{*}=\frac{-2 \beta N}{\psi \gamma},
$$

where $\psi$ is the root of $-3 e^{Z} Z^{2}+6 e^{Z} Z+e^{Z} Z^{3}-6 e^{Z}-$ $6 \mathcal{O}^{*}+6=0$ that minimizes (14).

\section{F. $T A S+M R C 2 \times 2$}

In this case TAS is employed at the transmitter $\left(N_{T R X}=1\right)$ and MRC is used at the receiver. The outage probability is [16]

$$
\mathcal{O}_{T A S(2 \times 2)}=1-2 e^{\frac{-\beta}{\rho}}\left(\frac{\beta}{\rho}+1\right)+e^{-2 \frac{\beta}{\rho}}\left(\frac{\beta}{\rho}+1\right)^{2},
$$

while the minimum required transmit power for $\mathcal{O}^{*}$ is

$$
\mathcal{P}_{T A S(2 \times 2)}^{*}=\frac{-\beta N}{\left[\mathcal{W}\left(-\left(1-\sqrt{\mathcal{O}^{*}}\right) e^{-1}\right)+1\right] \gamma} .
$$

\section{G. $B F+M R C 2 \times 2$}

The use of $\mathrm{BF}$ at the $\mathrm{BS}$, in combination with MRC at the $\mathrm{MS}$, is considered in the BF+MRC $2 \times 2$ scheme $\left(N_{T R X}=2\right)$. The outage probability becomes [18]

$$
\mathcal{O}_{B F(2 \times 2)}=1-e^{-\frac{\beta}{\rho}}\left(2+\frac{\beta^{2}}{\rho^{2}}\right)+e^{-2 \frac{\beta}{\rho}}
$$

while the minimum required transmit power for $\mathcal{O}^{*}$ is determined as

$$
\mathcal{P}_{B F(2 \times 2)}^{*}=\frac{-\beta N}{\Psi \gamma},
$$

where $\Psi$ represents the root of $2 e^{Z}-e^{2 Z}+\mathcal{O}^{*}-1+e^{Z} Z^{2}=0$ that minimizes (18).

\section{H. $V$-BLAST $2 \times 2$}

In a V-BLAST $2 \times 2$ system the information bit stream to be sent is split into two substreams and transmitted in parallel by the two BS antennas $\left(N_{T R X}=2\right)$. The received data is decoded in two detection steps [19]. Moreover, in order to obtain the same throughput of the previous methods, we consider that each transmit antenna in the V-BLAST scheme operates at half the rate utilized in the other methods.

The outage probability at the first detection step is [19]

$$
\mathcal{O}_{V B L A S T, F 1}=1-2 e^{\frac{-\beta^{\prime}}{\rho}}+\left(1+\frac{\beta^{\prime}}{2 \rho}\right) e^{-2 \frac{\beta^{\prime}}{\rho}},
$$

where $\beta^{\prime}=2^{\frac{\delta}{2}}-1$. The outage probability at the second detection step is [19]

$$
\mathcal{O}_{V B L A S T, F 2}=\mathcal{O}_{H}\left(2-\mathcal{O}_{H}\right),
$$

where $\mathcal{O}_{H}=1-e^{\frac{-\beta^{\prime}}{\rho}}\left(1+\frac{\beta^{\prime}}{\rho}\right)$. Then, the overall outage probability becomes

$\mathcal{O}_{V B L A S T}=\mathcal{O}_{V B L A S T, F 1}+\mathcal{O}_{V B L A S T, F 2}\left(1-\mathcal{O}_{V B L A S T, F 1}\right)$

As for relatively high SNR or sufficiently small outage probability values $\mathcal{O}_{V B L A S T} \simeq \mathcal{O}_{V B L A S T, F 1}$, the minimum transmit power for a target outage probability $\mathcal{O}^{*}$ can be approximated as

$$
\mathcal{P}_{V B L A S T}^{*} \simeq \frac{e^{2 \Phi} \beta N}{2 \gamma\left(2 e^{\Phi}-e^{2 \Phi}+\mathcal{O}^{*}-1\right)},
$$

where $\Phi$ is the root of $Z e^{2 Z}+4 e^{Z}-2 e^{2 Z}+2 \mathcal{O}^{*}-2=0$ that minimizes (22). It is important to remark that for the outage probability values considered in this paper the approximation $\mathcal{O}_{V B L A S T} \simeq \mathcal{O}_{V B L A S T, F 1}$ is very tight.

\section{RESUlts}

The performance of the transmission schemes is numerically evaluated in this section. The system parameters are: $N_{0}=$ $-174 \mathrm{dBm} / \mathrm{Hz}, B=10 \mathrm{MHz}, \alpha=3$. The parameters of the macro power model follow [8], with $P_{0}=84 \mathrm{~W}$ and $\Delta_{p}=2.8$. For increased efficiency, we consider that the macro BS uses a remote radio head (RRH), and as a consequence, the power amplifier module is mounted at the same physical 
TABLE I: Power Model Parameters

\begin{tabular}{c|c|c}
\hline BS type & $P_{0}[\mathrm{~W}]$ & $\Delta_{p}$ \\
\hline Macrocell $(\mathrm{RRH})$ & 84.0 & 2.8 \\
Microcell & 56.0 & 2.6 \\
Picocell & 6.8 & 4.0 \\
Femtocell & 4.8 & 8.0 \\
\hline
\end{tabular}

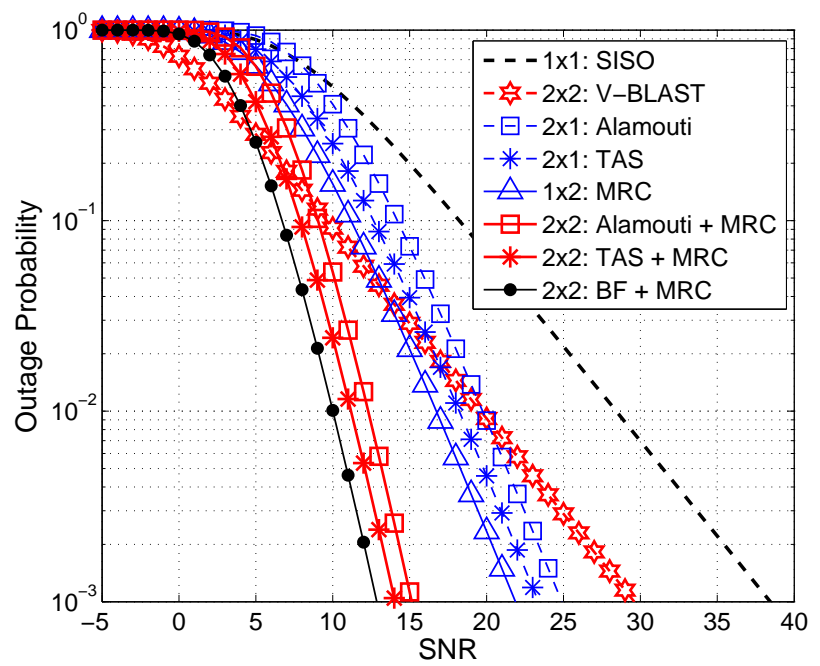

Fig. 1: Outage probability versus SNR for $\delta=3 \mathrm{~b} / \mathrm{s} / \mathrm{Hz}$.

location as its corresponding transmit antenna. Table I shows the power model parameters for other BS types.

Figure 1 illustrates the outage behavior of the considered transmission schemes. In general the SISO scheme presents the worst performance, while the $\mathrm{BF}+\mathrm{MRC} 2 \times 2$ requires the lowest SNR for most of the outage probabilities. Note also the difference in diversity order achieved by each scheme. However, as we show next, the best scheme in terms of outage probability is not necessarily the most energy efficient.

Figure 2 presents the consumed energy per bit for a target outage probability of $\mathcal{O}=10^{-2}$ and $\delta=3 \mathrm{~b} / \mathrm{s} / \mathrm{Hz}$, considering a macro BS with RRH. The SISO scheme is the least energy efficient one for most of the BS-MS distances. However, for short distances the transmitting circuitry consumption is a quite relevant factor, and SISO outperforms Alamouti 2x1, Alamouti+MRC 2x2, BF+MRC 2x2, and V-BLAST 2x2 for $d<455 \mathrm{~m}$. On the other hand, the combination of TAS with MRC with two transmit and two receive antennas (TAS+MRC 2x2) is the most energy efficient scheme for any BS-MS distance, outperforming other 2x2 MIMO schemes, as Alamouti+MRC $2 \times 2, B F+M R C 2 \times 2$, and V-BLAST $2 \times 2$. It is also important to note that the TAS scheme with only one receiving antenna (TAS $2 \times 1$ ) can outperform various MIMO $2 \times 2$ schemes. The BF+MRC $2 \times 2$ scheme is outperformed by TAS $2 \times 1$ for $d<1065 \mathrm{~m}$; the Alamouti+MRC $2 \times 2$ scheme is outperformed for $d<1115 \mathrm{~m}$, and the 2x2 V-BLAST scheme is outperformed for any distance. Thus, in this scenario the SISO scheme is the worst option for most of the distances and TAS+MRC $2 \times 2$ is the most energy efficient, as it takes

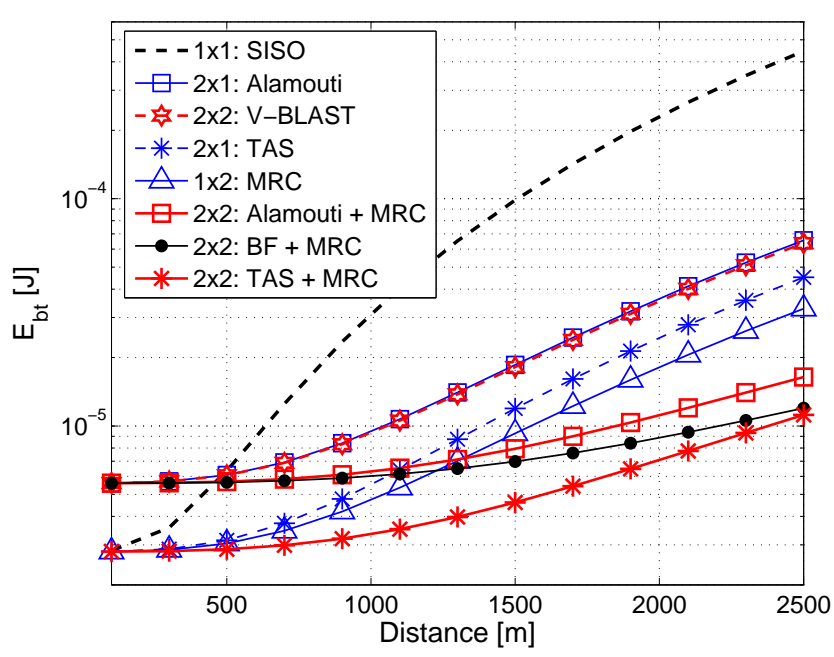

Fig. 2: Total consumed energy per bit considering the transmission from a macro BS for $\mathcal{O}=10^{-2}$, and $\delta=3 \mathrm{~b} / \mathrm{s} / \mathrm{Hz}$.

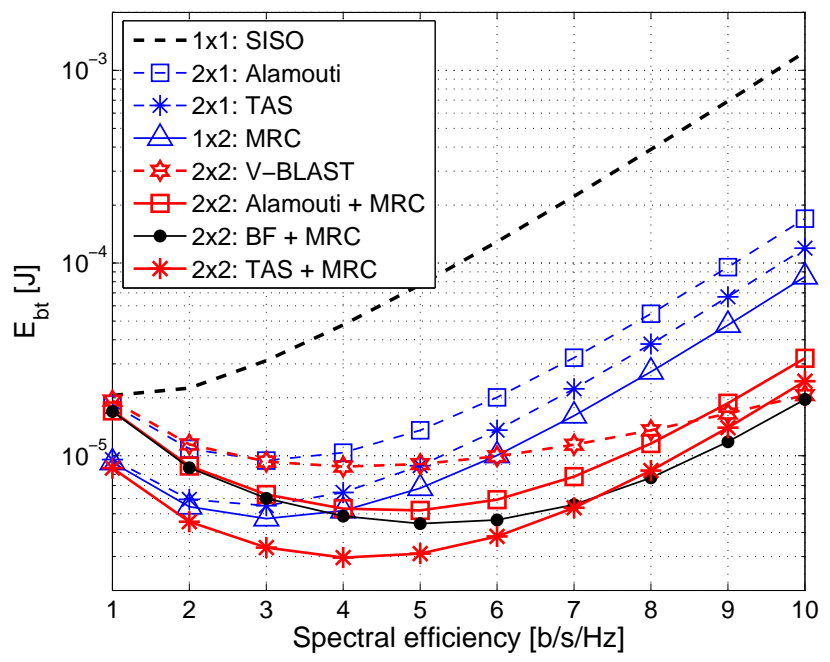

Fig. 3: Total consumed energy per bit considering the transmission from a macro BS for $\mathcal{O}=10^{-2}$, and $d=1000 \mathrm{~m}$.

advantage of the spatial diversity both in the transmission and in the reception, with lower transmit circuitry consumption if compared to the other MIMO $2 \times 2$ schemes. Finally, it is interesting to note that although the Alamouti+MRC $2 \times 2$ and the BF+MRC $2 \times 2$ schemes are among the best in terms of outage probability (from Figure 1), when the total energy consumption is considered, these schemes are outperformed by others that present worse outage performance.

Similar conclusions to those from Figure 2 can be obtained for other spectral efficiencies and the same $\mathcal{O}=10^{-2}$, as shown in Figure 3. Note that with a fixed BS-MS distance $d=1000 \mathrm{~m}$, the TAS+MRC $2 \times 2$ scheme is the most energy efficient for most of the spectral efficiencies, and is only outperformed by the BF+MRC $2 \times 2$ for $\delta>7 \mathrm{~b} / \mathrm{s} / \mathrm{Hz}$. 


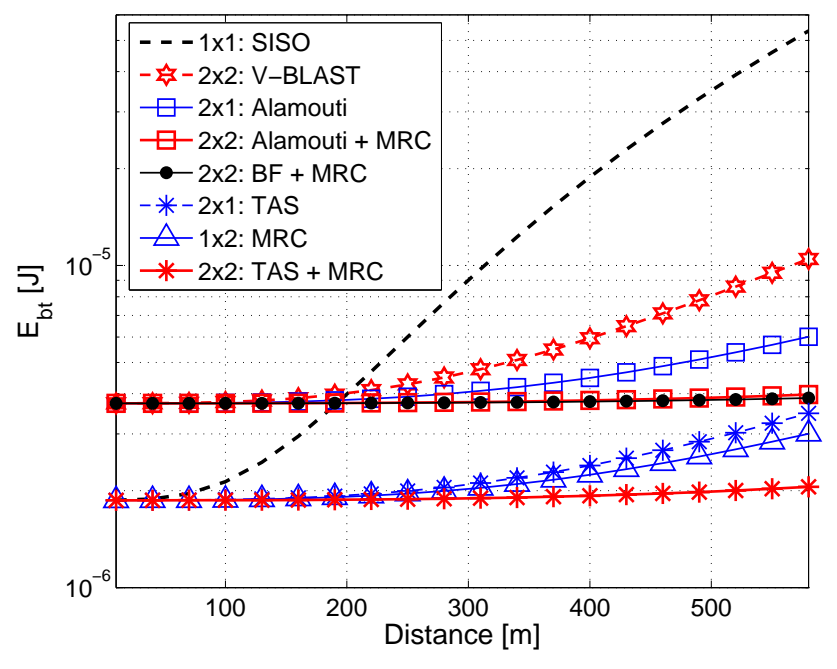

Fig. 4: Total consumed energy per bit considering the transmission from a micro BS for $\mathcal{O}=10^{-3}$, and $\delta=3 \mathrm{~b} / \mathrm{s} / \mathrm{Hz}$.

Furthermore, the SISO scheme is still the least energy efficient for any $\delta$, and the TAS $2 x 1$ scheme is outperformed by the other $2 \times 2$ schemes as the spectral efficiency increases.

In the case of a weaker outage probability requirement, as $\mathcal{O}=10^{-1}$, the transmitting circuitry consumption becomes even more relevant in the analysis, and TAS+MRC $2 \times 2$, MRC $1 \times 2$, and TAS $2 \times 1$ are the most energy efficient schemes for most of the distances, with TAS+MRC $2 \times 2$ being the most energy efficient of all. Moreover, although the SISO scheme is still the least energy efficient for most of the distances, its performance becomes more competitive. In opposition, for an increased outage probability requirement as $\mathcal{O}=10^{-3}$, although the relevancy of the transmitting circuitry consumption decreases, TAS+MRC $2 \times 2$ is still the most energy efficient scheme for most of the distances, being outperformed by BF+MRC 2x2 only when $d>2250 \mathrm{~m}$.

Figure 4 presents the consumed energy per bit in the case of a micro BS for $\mathcal{O}=10^{-3}$ and $\delta=3 \mathrm{~b} / \mathrm{s} / \mathrm{Hz}$. Again, the TAS+MRC $2 \times 2$ scheme is the most energy efficient, and TAS $2 \times 1$ can outperform the $2 \times 2$ MIMO schemes V-BLAST, $\mathrm{BF}+\mathrm{MRC}$, and Alamouti+MRC. Moreover, when compared to Figure 2, the advantage of TAS+MRC 2x2 over BF+MRC 2x2 increases considerably, so that the efficiency of TAS is even more evident. In case of a pico or femto BS the conclusions are generally the same, the TAS+MRC $2 \times 2$ scheme is the most energy efficient, while TAS $2 \times 1$ can outperform $\mathrm{BF}+\mathrm{MRC}$ $2 \times 2$, Alamouti+MRC $2 \times 2$, and V-BLAST $2 \times 2$.

\section{CONCLUSIONS}

We investigate the energy efficiency of MIMO systems for a given target outage probability, considering different types of base stations. We show that the combination of TAS and MRC, even though not the best one in terms of outage probability, can be a very energy efficient solution. That is a consequence of TAS requiring a single RF chain, while the other MIMO schemes require a RF chain per transmit antenna, considerably compromising their energy efficiency.

\section{ACKNOWLEDGEMENT}

This work was partially supported by CNPq and CAPES, Brazil (grant BEX 8642/11-7).

\section{REFERENCES}

[1] Z. Hasan, H. Boostanimehr, and V. K. Bhargava, "Green Cellular Networks: A Survey, Some Research Issues and Challenges," IEEE Communications Surveys \& Tutorials, vol.13, no.4, pp.524-540, Fourth Quarter 2011.

[2] S. Tombaz, M. Usman, and J. Zander, "Energy Efficiency Improvements Through Heterogeneous Networks in Diverse Traffic Distribution Scenarios," in 6th International ICST Conference on Communications and Networking in China - Chinacom11, Harbin, 2011.

[3] S. Tombaz, A. Vastberg, and J. Zander, "Energy- and Cost-Efficient Ultra-High-Capacity Wireless Access," in IEEE Wireless Networks, vol. 18, no. 5, pp. 18-24, October 2011.

[4] G. P. Fettweis and E. Zimmermann, "ICT Energy Consumption - Trends and Challenges," in Proceedings of the 11th International Symposium on Wireless Personal Multimedia Communications - WPMC'08, Lapland, September 2008.

[5] F. Richter, A. J. Fehske, G. P. Fettweis, "Energy Efficiency Aspects of Base Station Deployment Strategies for Cellular Networks," in IEEE Vehicular Technology Conference Fall (VTC 2009-Fall), Anchorage, 2009.

[6] H. Klessig, A. J. Fehske, G. P. Fettweis, "Energy Efficiency Gains in Interference-Limited Heterogeneous Cellular Mobile Radio Networks with Random Micro Site Deployment," in IEEE 34th Sarnoff Symposium, Princeton, 2011.

[7] O. Onireti, F. Heliot, and M. A. Imran, "On the Energy-EfficiencySpectral Efficiency Trade-off in the Uplink of CoMP System," IEEE Transactions on Wireless Communications, vol. 11, no. 2, pp.556-561, February 2012.

[8] G. Auer, V. Giannini, I. G'odor, P. Skillermark, M. Olsson, M. A. Imran, D. Sabella, M. J. Gonzalez, C. Desset, O. Blume, A. Fehske, "How Much Energy is Needed to Run a Wireless Network?", IEEE Communications Magazine, vol. 18, no. 5, pp. 40-49, October 2011.

[9] S. Cui, A. Goldsmith, A. Bahai, "Energy-Efficiency of MIMO and Cooperative MIMO Techniques in Sensor Networks," IEEE Journal on Selected Areas in Communications vol. 22, no. 6, pp. 1089-1098, 2004.

[10] F. D. Cardoso, and L. M. Correia, "MIMO Gain and Energy Efficiency in LTE," 2012 IEEE Wireless Communications and Networking Conference: Mobile and Wireless Networks - WCNC 2012, Paris, 2012.

[11] F. Heliot, M. A. Imran, and R. Tafazolli, "On the energy efficiency gain of MIMO communication under various power consumption models," Future Network \& Mobile Summit (FutureNetw) 2011, vol., no., pp.1-9, 15-17 June 2011.

[12] M. Kakitani, G. Brante, R. Souza, and M. Imran, "Energy Efficiency of Transmit Diversity Systems Under a Realistic Power Consumption Model," IEEE Communications Letters, vol.17, no.1, pp.119-122, January 2013.

[13] A. Goldsmith, Wireless Communications. New York, NY, USA: Cambridge University Press, 2005.

[14] T. M. Cover, J. A. Thomas, Elements of Information Theory, 2nd ed., John Wiley \& Sons, 1991.

[15] H. Lee, R. Heath, E. Powers, "Information outage probability and diversity order of Alamouti transmit diversity in time-selective fading channels,'IEEE Trans. Veh. Technol., vol. 57, no. 6, pp. 3890 -3895, November 2008

[16] Z. Chen, J. Yuan, B. Vucetic, "Analysis of transmit antenna selection/maximal-ratio combining in Rayleigh fading channels," IEEE Trans. Veh. Technol., vol. 54, no. 4, pp. 1312 - 1321, July 2005.

[17] M. K. Simon and M. S. Alouini, Digital Communication over Fading Channels. Wiley Interscience, 2004.

[18] A. J. Grant, "Performance analysis of transmit beamforming," IEEE Trans. Commmun., vol. 53, no. 4, pp. 738 - 744, April 2005.

[19] S. Loyka and F. Gagnon, "Performance analysis of the V-BLAST algorithm: an analytical approach," IEEE Trans. Wireless Commun., vol. 3, no. 4, pp. 1326 - 1337, July 2004. 